\title{
FEATURES OF EVALUATION DRIFT EFFECT DURING KEY COMPARISON COOMET.EM-K5
}

\author{
STANISLAV KARPENKO \\ State Enterprise «Ukrmeterteststandart» \\ 4, Metrologichna Str., 03143, Kyiv, Ukraine, Tel./Fax: +38 04452615 41, \\ e-mail: s.r.karpenko86@gmail.com
}

\begin{abstract}
Results of pilot laboratory and participating laboratories which are measuring the same travelling standard during Key Comparison must be most similar. However, this may not always be possible, because the travelling standard which is sent in turn to all participating laboratories is not always perfectly stable. So that it is necessary to take into account the drift effect of the travelling standard. The article describes some problems concerning the evaluation drift effect of travelling standard and its further influence on evaluation key comparison reference value during Key Comparison. The procedure for evaluation drift effect when its model can be linear or not linear was presented. Comparative analysis of the proposed procedures for evaluation drift effect is applied to the Key Comparison of Power COOMET.EM-K5 was done.
\end{abstract}

Keywords: drift effect, key comparison, electric power, key comparison reference value, travelling standard.

\section{Introduction}

Key comparisons (KC) are the special interlaboratory comparisons for National Metrology Institutes (NMIs) and regional metrology organizations (RMOs) around the world which are carried out within the framework of the International Committee for Weights and Measures (CIPM) Mutual Recognition Arrangement (MRA) [1]. The main purpose of $K C$ is the determination of the equivalence between laboratories of different NMIs.

The unilateral degree of equivalence of a laboratory is obtained as the deviation of its measurement result from the KC reference value (KCRV), together with the uncertainty associated with this deviation according to the MRA [2].

The MRA describes in general how the data of KC should be evaluated but it does not provide enough specifics to define an unambiguous analysis. Consequently many different ways of evaluating $\mathrm{KC}$ data have been suggested over the years [3].

Ideally, all laboratories of different NMls participating in a KC are measuring the same travelling standard (TS), which makes the comparison of reported results most meaningful. However, this may not always be possible, because the TS that is sent in turn to all laboratories common TS is not always stable and may change its value over time in the course of the $\mathrm{KC}$. If in planning the $\mathrm{KC}$ the circular scheme will be used or a small number of participating laboratories then for a stable TC, checks can make on the unvarying value in a pilot laboratory at the start and at the end of the KC. In such a way the pilot laboratory makes measurements at least twice [4]. But if in planning the KC the radial scheme will be used the TS is returned to the pilot laboratory after each measurement at a participating laboratory or more often after performing a group of measurements at participating laboratories. During the analysis of the KC data then needs to account for these drift effects.

It should be noted that the accuracy of calculation of KCRV depends on accuracy of determining drift effects during KC [5-7]. Nevertheless since KC are utilized to approve, or disapprove, the calibration and measurement capabilities (CMCs) quoted by NMls they have received much interest over the years [8].

\section{General remark on Key Comparison of Power COOMET.EM-K5}

To support the CMCs declared by members of COOMET in the framework of the CIPMMRA, SE «Ukrmetrteststandart» (UMTS, Ukraine) organized COOMET KC of electric power unit for electrical standards of low-frequency $50 / 60 \mathrm{~Hz}$ power. UMTS was proposed to be the pilot laboratory, which would be responsible for providing the TS, coordinating the schedule, collecting and analyzing the comparison data, and preparing the draft report. 
National Institute of Metrology (NIM, China) and D.I. Mendeleyev institute for Metrology (VNIIM, Russia) were proposed to be the linking NMls for the linking process between CCEM-K5 KC and COOMET.EM-K5 KC [9]. TS of low-frequency 50/60 Hz power will be compared at $11 \mathrm{NMIs}$ from COOMET and EURAMET to establish the relationship between the electrical units of AC power at these laboratories of different NMIs. COOMET.EM-K5 KC will be linked to CCEM-K5 KC.

\section{Evaluation of the travelling standard's drift effect}

During $\mathrm{KC}$ all the participating laboratories should measure the identical TS, which often is not perfectly stable. So that it is necessary to take into account the instabilities arising from the ageing of the TS or by several physical or mechanical changes during the transportation process or from the time. The presence of drift of TS, directly influences the quality of KC.

If preliminary analysis of the $\mathrm{KC}$ data indicates the drift in the measured value of the TS, it is necessary to evaluate this drift for subsequent use in establishing the KCRV for the KC and calculating the degrees of equivalence of the NMls standards.

The pilot laboratory performed repeated measurements over the duration of the $\mathrm{KC}$ in order to monitor the stability of the TS and also the correction to compensate the drift can be evaluated. The evaluated correction which depends on both the stability of the TS and the long term stability of the measurements and its standard uncertainty will be included in the model describing the measurement process of the KC [10]. I applied the different approaches for evaluation drift effect to the COOMET.EM-K5 KC [9].

During the COOMET.EM-K5 KC, the TS RD-33-332 (serial number 301308) is measured at the pilot laboratory (UMTS) for each time after $i$ participating laboratory made measurements. Also before the beginning COOMET.EM-K5 KC the pilot laboratory (UMTS) researched the TS for the drift effects. The measurement data, which are measurement results (mean values) by UMTS are listed in Table 1 . In this example, I consider only the measurement point $120 \mathrm{~V}, 5 \mathrm{~A}, \mathrm{PF} 1.0,50 \mathrm{~Hz}$.

Table 1. Measurement data

\begin{tabular}{|c|c|c|c|c|c|c|c|c|c|c|c|}
\hline \multirow[t]{5}{*}{ № } & \multicolumn{10}{|c|}{ Measurement points } & \multirow{5}{*}{$\begin{array}{c}\text { Period } \\
\text { in } \\
\text { weeks }\end{array}$} \\
\hline & \multicolumn{5}{|c|}{$120 \mathrm{~V}, 5 \mathrm{~A}, \mathrm{PF}, 50 \mathrm{~Hz}$} & \multicolumn{5}{|c|}{$120 \mathrm{~V}, 5 \mathrm{~A}, \mathrm{PF}, 53 \mathrm{~Hz}$} & \\
\hline & 1.0 & 0.5 & 0.5 & 0.0 & 0.0 & 1.0 & 0.5 & 0.5 & 0.0 & 0.0 & \\
\hline & & Lag & Lead & Lag & Lead & & Lag & Lead & Lag & Lead & \\
\hline & \multicolumn{10}{|c|}{ Measurement results, $\mu \mathrm{W} / \mathrm{VA}$} & \\
\hline UMTS1 & 1.8 & 2.5 & -4.6 & 3.8 & -4.0 & 0.9 & 1.9 & -4.3 & 3.3 & -3.9 & 0 \\
\hline UMTS2 & 1.8 & 2.4 & -4.6 & 3.7 & -4.1 & 1.0 & 1.9 & -4.3 & 3.3 & -4.0 & 2 \\
\hline UMTS3 & 1.9 & 2.5 & -4.5 & 3.8 & -4.1 & 0.9 & 1.8 & -4.4 & 3.2 & -4.0 & 5 \\
\hline UMTS4 & 1.8 & 2.3 & -4.4 & 3.9 & -3.9 & 1.1 & 1.9 & -4.2 & 3.3 & -3.9 & 7 \\
\hline UMTS5 & 1.9 & 2.5 & -4.5 & 3.7 & -4.1 & 0.9 & 1.9 & -4.4 & 3.4 & -3.8 & 12 \\
\hline UMTS6 & 1.7 & 2.4 & -4.4 & 3.8 & -4.0 & 1.1 & 2.0 & -4.3 & 3.3 & -4.0 & 24 \\
\hline
\end{tabular}

\subsection{Linear drift}

Sometimes the data can show the linear drift, such cases have been considered by different models [4-6].

If there are no regularities that could be the basis of a drift model, one usually is given by a proposed linear model:

$$
x-\bar{x}=m_{D R I F T} \cdot\left(y_{i}-\bar{y}\right),
$$

where $y_{i}$ (month) - the given date; $\bar{y}$ (month) - the average date of the NMI measurements on the TS; $x(\mu \mathrm{W} / \mathrm{VA})$ - the measured value given by the linear drift on date $y_{i} ; \bar{x}(\mu \mathrm{W} / \mathrm{VA})-$ the average measured value of NMI measurements on the TS; $m_{D R I F T}(\mu \mathrm{W} / \mathrm{VA} / \mathrm{month})$ - the drift of the measured value per month. The proposed procedure of evaluated linear drift is illustrated by its application to recent KC COOMET.EM-K5.

After analyzing measurements were specified that the behavior of TS is the linear drift and can be seen in Figure 1. Also for evaluation of linear drift of TS can be used linear approximations or $1^{\text {st }}$ order polynomial regression. 


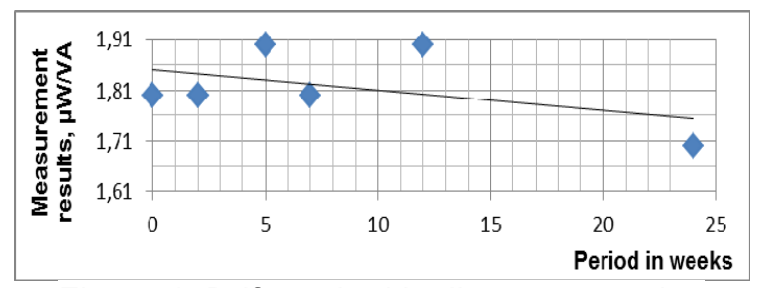

Figure 1. Drift tracked by linear regression

\subsection{Non-linear drift}

Nevertheless, in most cases the TS shows non-linear drifts, which are probably caused by mechanical changes arising from the transport or from the time.

In such cases the evaluation of the uncertainty due to the instability could be determined from the standard deviation of the measurements of the linking laboratory. And the standard deviation of the measurements of the linking laboratory would be considered in the budget uncertainty of each participating laboratory in KC [9]. To estimate non-linear drifts in the TS during COOMET.EM-K5 KC are proposed to use procedure of evaluation the polynomial regression or piece-wise linear approximations. The polynomial regression model is:

$$
y_{i}=a_{0}+a_{1} x_{1}+a_{2} x_{2}^{2}+a_{3} x_{3}^{3}+\ldots \ldots . .+a_{n} x_{n}^{n}+\xi
$$

where $\xi=$ random error with zero mean.

It was assumed that the TS drift in non-linear fashion. Researched non-linear effects are caused probably by mechanical changes during the transportation process.

To estimate non-linear drift in the TS, a polynomial regression was fitted to the six UMTS measurements for each power factor (PF). But the numbers of UMTS measurements will be equal the list of dates of measurements in according to the Technical Protocol [8]. Polynomial regression was selected to track the drift behavior of the TS (Table 2). Also was calculated the determination coefficient $\left(R^{2}\right)$. Applying the Chaddock scale the determination coefficient $\left(R^{2}\right)$ gets quality characteristic which are needed to assess the quality of the selection of the regression equation.

Table 2. Selected polynomial regression to track the drift behavior of the TS

\begin{tabular}{|c|c|c|c|c|}
\hline $\begin{array}{l}\text { Polynomial } \\
\text { regression }\end{array}$ & Regression equation & $\begin{array}{l}\text { Determi- } \\
\text { nation } \\
\text { coefficient } \\
\left(R^{2}\right)\end{array}$ & $\begin{array}{c}\text { Quality } \\
\text { characteristic } \\
\text { according } \\
\text { Chaddock } \\
\text { scale }\end{array}$ & Conclusion \\
\hline $1^{\text {st }}$ order & $y_{U M T S}=-0.004 \cdot t(U M T S)+1.8502$ & 0.2176 & Weak level & Don't use \\
\hline $2^{\text {nd }}$ order & $\begin{array}{l}y_{U M T S}=-0.0009 \cdot t(U M T S)^{2}+0.0173 \\
\cdot t(U M T S)+1.7878\end{array}$ & 0.7161 & Salient level & Not good \\
\hline $3^{\text {rd }}$ order & $\begin{array}{l}y_{U M T S}=-0.00005 \cdot t(U M T S)^{3}+0.0007 \\
\cdot t(U M T S)^{2}+0.0054 \cdot t(U M T S)+1.8005\end{array}$ & 0.7430 & High level & $\begin{array}{l}\text { Recommen- } \\
\text { ded }\end{array}$ \\
\hline $4^{\text {th }}$ order & $\begin{array}{l}y_{\text {UMTS }}=-0.00002 \cdot t(\text { UMTS })^{4}+0.0008 \\
\cdot t(U M T S)^{3}-0.0089 \cdot t(U M T S)^{2}+ \\
+0.0381 \cdot t(U M T S)+1.7885\end{array}$ & 0.7783 & High level & Good \\
\hline $5^{\text {th }}$ order & $\begin{array}{l}y_{\text {UMTS }}=-0.00003 \cdot t(\text { UMTS })^{5}+0.00012 \cdot \\
\cdot t(\text { UMTS })^{4}-0.0165 \cdot t(\text { UMTS })^{3}+0.0806 \cdot \\
\cdot t(\text { UMTS })^{2}-0.1044 \cdot t(\text { UMTS })+1.8000\end{array}$ & 1.0000 & $\begin{array}{l}\text { Functional } \\
\text { dependence } \\
\text { between } \\
\text { variables }\end{array}$ & Very well \\
\hline
\end{tabular}

The $2^{\text {nd }}-5^{\text {th }}$ order polynomial regressions can be seen in Figures $2-5$. The drift of the TS is calculated according to the formula:

$$
\operatorname{DRIFT}(T S)=\frac{1}{n} \sum_{i=1}^{n} x_{\text {icorr.pol }}-\frac{1}{m} \sum_{j=1}^{m} x_{\text {jmeas.val }}=\bar{x}_{\text {corr.pol }}-\bar{x}_{\text {meas.val }},
$$

where $x_{\text {icorr.pol }}-i$ corrected measurement made by pilot laboratory; $x_{\text {jmeas.val }}-j$ measurement made by pilot laboratory; $\bar{x}_{\text {corr.pol }}-$ average corrected measurement result; $\bar{x}_{\text {meas.val }}-$ average measurement value made by pilot laboratory. 


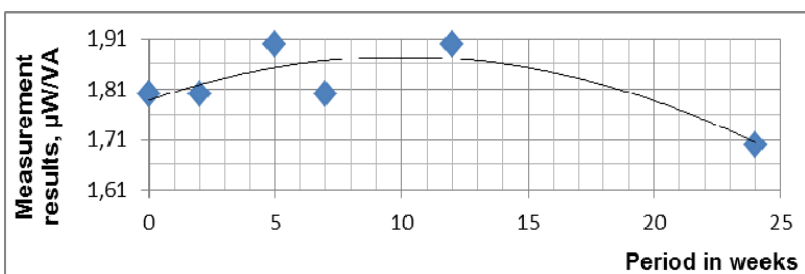

Figure 2 . The drift tracked by $2^{\text {nd }}$ order polynomial regression

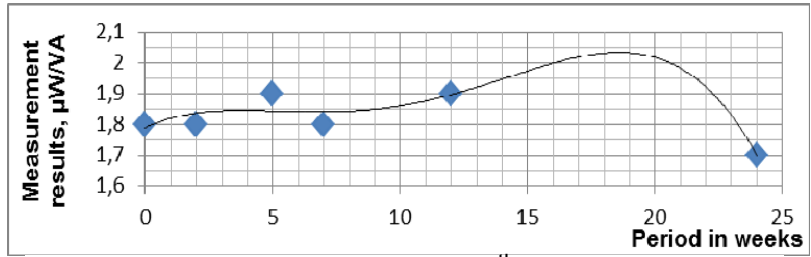

Figure 4 . The drift tracked by $4^{\text {th }}$ order polynomial regression

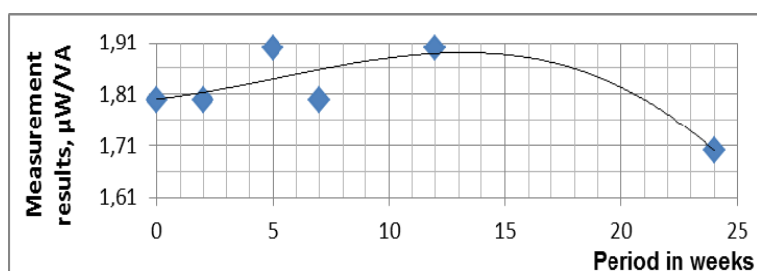

Figure 3. The drift tracked by $3^{\text {rd }}$ order polynomial regression

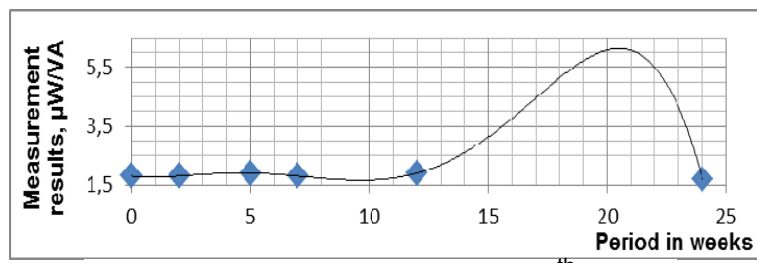

Figure 5 . The drift tracked by $5^{\text {th }}$ order polynomial regression

Ideally, I consider the drift of the TS have to be evaluated for each measurement of pilot laboratory in a KC individually for each participating laboratory and sum of them. For evaluation drift of the TS was proposed the next formula:

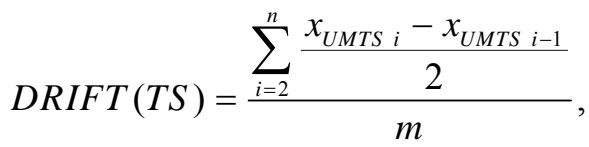

where $x_{\text {UMTS } i}-i$ measurement made by pilot; $x_{\text {UMTS } i-1}-i-1$ measurement made by pilot; $m$ the total measurement period of pilot laboratory.

Comparative analysis of the proposed procedures for evaluation drift when the model is linear or not-linear which is applied to the KC of Power COOMET.EM-K5 was done (Table 3).

Table 3. Evaluated drift of the TS

\begin{tabular}{|c|c|c|c|c|c|c|c|}
\hline \multirow{3}{*}{$\begin{array}{l}\text { Evaluated } \\
\text { drift of the } \\
\text { travelling }\end{array}$} & \multicolumn{7}{|c|}{ Model of drift of the travelling standard } \\
\hline & \multirow{3}{*}{ near } & \multicolumn{6}{|c|}{ Non-linear } \\
\hline & & \multicolumn{5}{|c|}{ ynomial regression } & \multirow{2}{*}{$\begin{array}{l}\text { Proposed } \\
\text { method }\end{array}$} \\
\hline standard, & & & $2^{\text {nd }}$ order & & $4^{\text {th }}$ order & $5^{\text {th }}$ or & \\
\hline$\mu \mathrm{W} / \mathrm{VA}$ & 0.026267 & 0.026267 & -0.000413 & 0.000156 & 0.016231 & -0.026713 & 0.002083 \\
\hline
\end{tabular}

\section{Conclusion}

The procedures for revealing and evaluating of the linear or non-linear drift effect of the TS when making KC of Power COOMET.EM-K5 was proposed. If the TS which is sent in turn to all participating laboratories is perfectly stable it is enough to apply linear drift model (linear approximations or $1^{\text {st }}$ order polynomial regression). However the TS is not perfectly stable than must be used non-linear drift model. Proposed procedure for evaluation nonlinear drift is easier in calculation part but may lose in accuracy in some cases.

\section{References}

[1] BIPM 1999. Mutual Recognition of National Measurement Standards, of Calibration and Measurement Certificates issued by National Metrology Institutes (CIPM revision 2003).

[2] Wübbeler G, Bodnar O, Mickan B and Elster C 2015 Explanatory power of degrees of equivalence in the presence of a random instability of the common measurand Metrol. 52 pp. 400-405.

[3] Elster C and Toman B 2013 Analysis of key comparison data: critical assessment of elements of current practice with suggested improvements Metrol. 50 pp. 549-555.

[4] Stepanov A V 2007 General problems of metrology and measurement technique - Comparing algorithms for evaluating key comparisons with linear drift in the reference standard, Meas. Tech. 50 pp. 1019-1027.

[5] Zhang N F, Liu H-K, Sedransk N and Strawderman W E 2004 Statistical analysis of key comparisons with linear trends Metrol. 41 pp. 231-237.

[6] Zhang N F, Strawderman W E, Liu H-K and Sedransk N 2006 Statistical analysis for multiple artifact problem in key comparisons with linear trends Metrol. 43 pp. 21-26.

[7] Elster C, Woger W, Cox M 2005 Analysis of key comparison data: unstable travelling standards Meas. Tech.48 pp. 883-893.

[8] Wübbeler G, Bodnar O and Elster C 2016 Bayesian hypothesis testing for key comparisons Metrologia 53 pp. 1131-1138.

[9] TECHNICAL PROTOCOL on COOMET 695/UA/16 Key Comparison of Power (COOMET.EM-K5). O. Velychko, S. Karpenko. - January 2017. SE "Ukrmeterteststandard" p. 15.

[10] Bergoglio M, Malengo A and Mari D 2011 Analysis of interlaboratory comparisons affected by correlations of the reference standards and drift of the travelling standards Measurement. 44 pp. 1461-1467. 